\title{
Computer Simulation of Volume Shrinkage after Mixing Container Media Components
}

\author{
Silvia Burés ${ }^{1,2}$, Franklin A. Pokorny ${ }^{1}$, David P. Landau ${ }^{2}$, and Alan M. Ferrenberg ${ }^{2,3}$ \\ University of Georgia, Athens, GA 30602 \\ Additional index words. substrates, artificial media, packing, pore size, porosity, soil physics, Monte Carlo, computer \\ programming

\begin{abstract}
A FORTRAN computer program was developed to simulate packing of spherical particles via a Monte Carlo procedure. Shrinkage in volume upon mixing different particle sizes was studied and simulated results were compared with experimental data. Maximum experimental shrinkage was obtained when the proportion of coarse particles of pine bark and sand mixtures ranged from $50 \%$ to $70 \%$ of the volume. Experimental shrinkage of a mixture of coarse and fine sand was closely reproduced by means of simulation. Particle size distribution appears to be the most important factor in relation to shrinkage and also in the establishment of relationships between the simulated and the experimental system.
\end{abstract}

Container media are essentially mixtures of different-sized particles. Physical properties of container media depend on the packing characteristics of particles, which are affected directly by their size distribution. Thus, to obtain container substrates with optimal properties, components are mixed in various proportions (Handreck, 1983; Richards et al., 1986). When materials of different particle sizes are mixed, fine particles fill the pores between the large particles, resulting in reduction of the final volume of the mixture in relation to the original volume of the separate materials (shrinkage). Thus, total porosity of the medium is decreased (Dallavalle, 1948). The study of volume variation upon mixing has practical applications in diverse fields (Haughey and Beveridge, 1969) and has been characterized for mixtures of various materials (Bodman and Constantin, 1965; Sohn and Moreland, 1968; Westman and Hugill, 1930). Degree of shrinkage depends on the relative size of particles that are mixed and on particle size distribution (Sohn and Moreland, 1968; Westman, 1936).

Shrinkage, as applied to container media, was studied by Spomer (1974), who defined the "threshold proportion" as that proportion of ingredients of different sizes that produces a mixture with minimum porosity. Several attempts have been made thereafter to determine and model shrinkage of artificial soil mixtures (Burés and Pokorny, 1991; Nash and Pokorny, 1990).

Container media modeling should be based on knowledge of the basic phenomena that occur at the particle level. Thus, fundamental research is necessary to understand the physical response of container medium systems. The possibility of devising a threedimensional simulation model that would provide some insight on volumetric interactions between container medium components arose from our previous research with a computer simulation program that packs particles in two dimensions; this program allows for the study of porosity variation when two particle sizes are mixed (Burés et al., 1993).

Computer simulation of density variation of binary mixtures of

Received for publication 4 Sept. 1992. Accepted for publication 4 Mar. 1993 Supported by state and Hatch funds allocated to the Georgia Experiment Station. We acknowledge Larry T. West, Crop and Soil Sciences Dept., Univ. of Georgia, for his valuable assistance in particle size and shape characterization, and Mark A. Farmer, Center for Ultrastructural Research, Univ. of Georgia, for very helpful discussions. The cost of publishing this paper was defrayed in part by the payment of page charges. Under postal regulations, this paper therefore must be hereby marked advertisement solely to indicate this fact.

${ }^{1}$ Dept. of Horticulture.

${ }^{2}$ Center for Simulational Physics.

${ }^{3}$ Univ. Computing and Networking Services. spheres as a function of the volume fraction of large particles was proposed by Visscher and Bolsterli (1972). One of the main problems these researchers encountered was the excessive computer time needed to pack many small particles in relation to the large particles in the mixture, which made the program rather inefficient. Davis and Carter (1990) proposed a reduced-dimension algorithm that gives an approximation of maximum shrinkage. None of these approaches, though, reproduces actual shrinkage when materials with unequal particle size distributions are mixed.

The objectives of this research were to a) develop a computer program that packs diverse size distributions of spherical particles in three dimensions, b) simulate shrinkage when mixing differentsized particles, c) study factors that affect shrinkage, and d) compare simulated results with experimental data.

\section{Materials and Methods}

Laboratory experiments. Theseexperiments usedcoarse $(0.841$ to $1 \mathrm{~mm})$ and fine $(0.420$ to $0.600 \mathrm{~mm})$ pine bark and sand. Bulk density of the four materials was obtained by packing $\approx 90 \mathrm{ml}$ of sample using a wrist-action shaker for $20 \mathrm{~min}$ (Pokorny and Henny, 1984). Once the bulk density of the four materials was known, we determined the weight of each component required to achieve additive bulk volumes of $100 \%$ from bulk densities of the single materials and calculated all possible mixtures of coarse and fine materials in 10\% volume increments (Nash and Pokomy, 1990). Volumes obtained after shaking for $20 \mathrm{~min}$ were measured for each of the mixtures in $10 \%$ increments of the four binary coarse and fine combinations. This method allowed us to calculate the difference between additive volume and final mixture volume, which is necessary for shrinkage determination.

A time-course experiment was conducted with fine sand to determine porosity (volume of pores $\times 100 /$ total bulk volume of container medium system) variation versus shaking time. For the binary mixture of coarse sand and fine sand, the volume also was determined before shaking (loose sample) to characterize effects of packing on shrinkage.

We measured shape factor and actual radius distributions of pine bark and sand with an image analyzer (CUE-2; Olympus, Southern Micro Instruments, Atlanta) and calculated the cumulative probability distribution of radii for the four materials.

Monte Carlo simulation. A FORTRAN computer program was written that packs spheres of one or two sizes or size distributions inside a container that has periodic boundary conditions in the two 
directions perpendicular to the gravitational field. Particles are initially situated randomly within the container and then are settled via a Monte Carlo procedure (Burés et al., 1993). Maximum allowed displacement per move was $0.1 \mathrm{~mm}$, with the length and direction of movement chosen at random (we use $\mathrm{mm}$ as the dimension on the simulations because it establishes an analogy with experimental measures). Particle overlapping was not allowed. This program can read two cumulative probability distributions of radii.

The system's volume was calculated by dividing the horizontal section of the container into 10,000 squares and determining the height of the uppermost sphere within each square.

The variation of porosity with Monte Carlo time also was studied (one Monte Carlo step is one attempt to move each of the particles downwards). We simulated the binary combinations of monosize spheres with diameters equal to the mean screen openings $(0.460$ and $0.255 \mathrm{~mm})$. Large and small spheres were mixed in $10 \%$ increments by calculating the actual bulk volume of spheres that should be mixed to obtain an additive volume of $100 \%$. Porosity is constant for any given uniform-sized system of spheres and does not depend on the particle size; however, for a small number of particles, porosity depends on the size of the container, and it was necessary to account for these size effects, because we often had to work with small systems of spheres to keep the program operating efficiently. We considered the bulk volume :particle volume ratio to be constant. In mixing bulk volumes of large and small particles, we actually mixed particle volumes and tested the resultant bulk volumes for equality of means with a $t$ test to account for effects of system size. The simulation was repeated using the average radii of sand particles and also with the actual distribution of particle sizes for sand.

Shrinkage curves. Shrinkage for experimental and simulated systems was determined from the difference between the additive volume of components and final mixture volume for any given mixture, divided by the additive volume of the components. Curves were developed where percentage shrinkage is expressed in relation to the percentage of the coarse component of a mixture.

The standard error of the mean for any mixture combination was calculated as a function of the standard errors of the volume at a given proportion of components and the standard errors of the volume calculation for large (coarse) and small (fine) particles by themselves.

\section{Results and Discussion}

The shrinkage curves from laboratory experiments (Fig. 1) show different maxima for the four binary combinations of coarse and fine pine bark and sand. Maxima are obtained when coarse materials are added in proportions that range from $50 \%$ to $70 \%$ by volume. Except for the mixture of coarse and fine pine bark (Fig. 1b), all curves are shifted to the right, which agrees with published data on mixtures of spheres or quasi-spherical particles (Sohn and Moreland, 1968; Westman, 1936). The small differences between the curves, taking into account that the method (size of the system, shaking time) was held constant, can be attributed to either intrinsic properties of the particular materials (shape, surface factors, composition) that can affect packing, or to particle size. Although pine bark and sand were sieved through the same sieves, actual size distribution and specific particle shape allows different-shaped particles to pass through a sieve, depending on their intermediate dimensions in relation to the mesh size (Allen, 1985).

The average shape factors were 0.77 (coarse) and 0.76 (fine) for sand, and 0.72 (coarse) and 0.69 (fine) for pine bark (Fig. 2). Although the modal value of the distribution was the same for the four materials (0.78), pine bark has a broader shape distribution (Fig. 2c and d). With the aid of a scanning electron microscope, particle shapes can be distinguished visually: pine bark particles range from fibers and rods to almost spheres, whereas sand

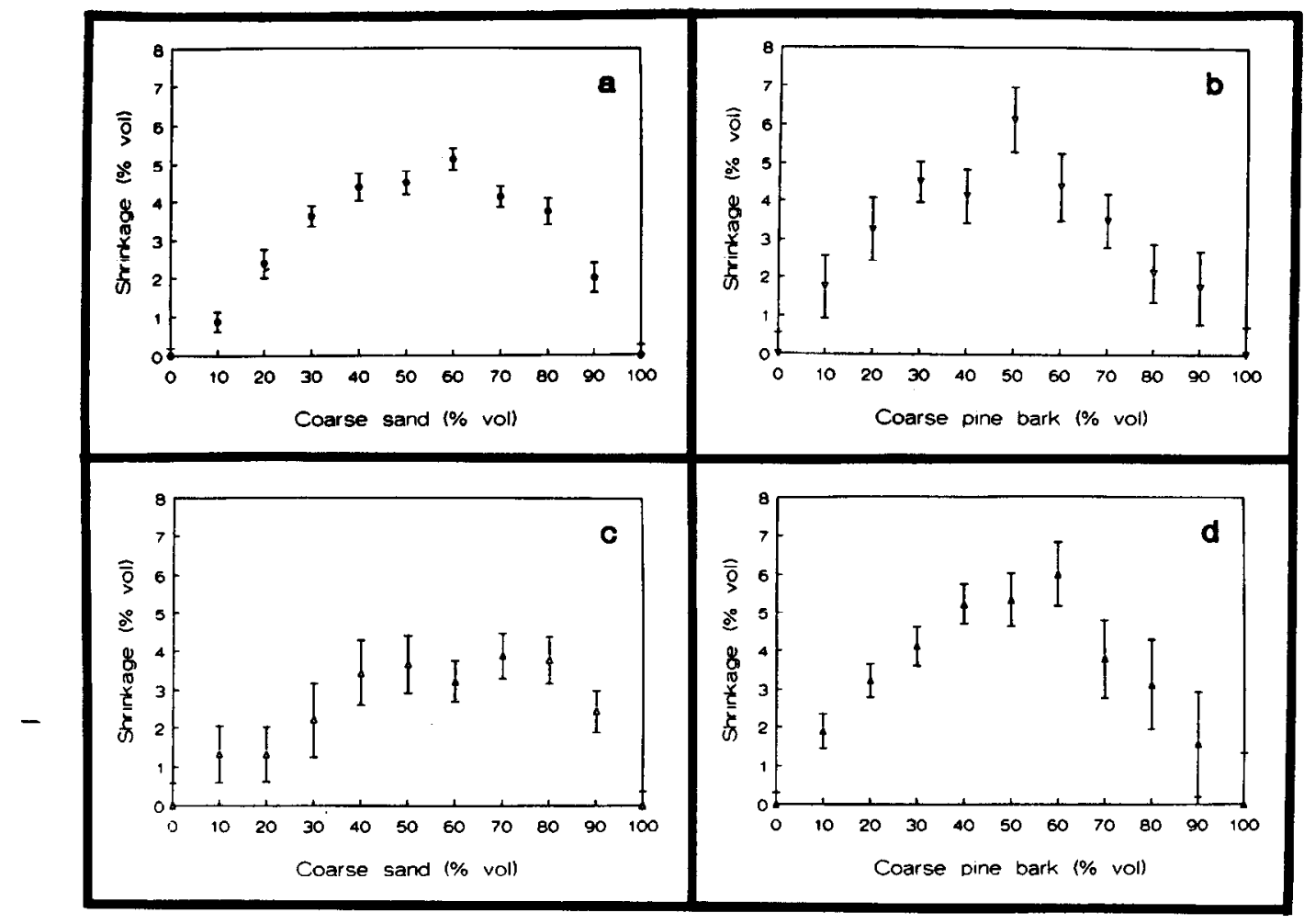

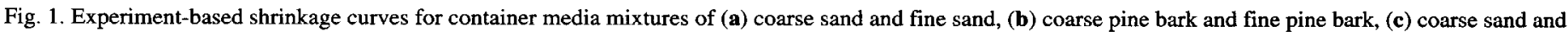
fine pine bark, and (d) coarse pine bark and fine sand. Coarse, 0.841 to $1.000 \mathrm{~mm}$, and fine, 0.420 to $0.600 \mathrm{~mm}$. 


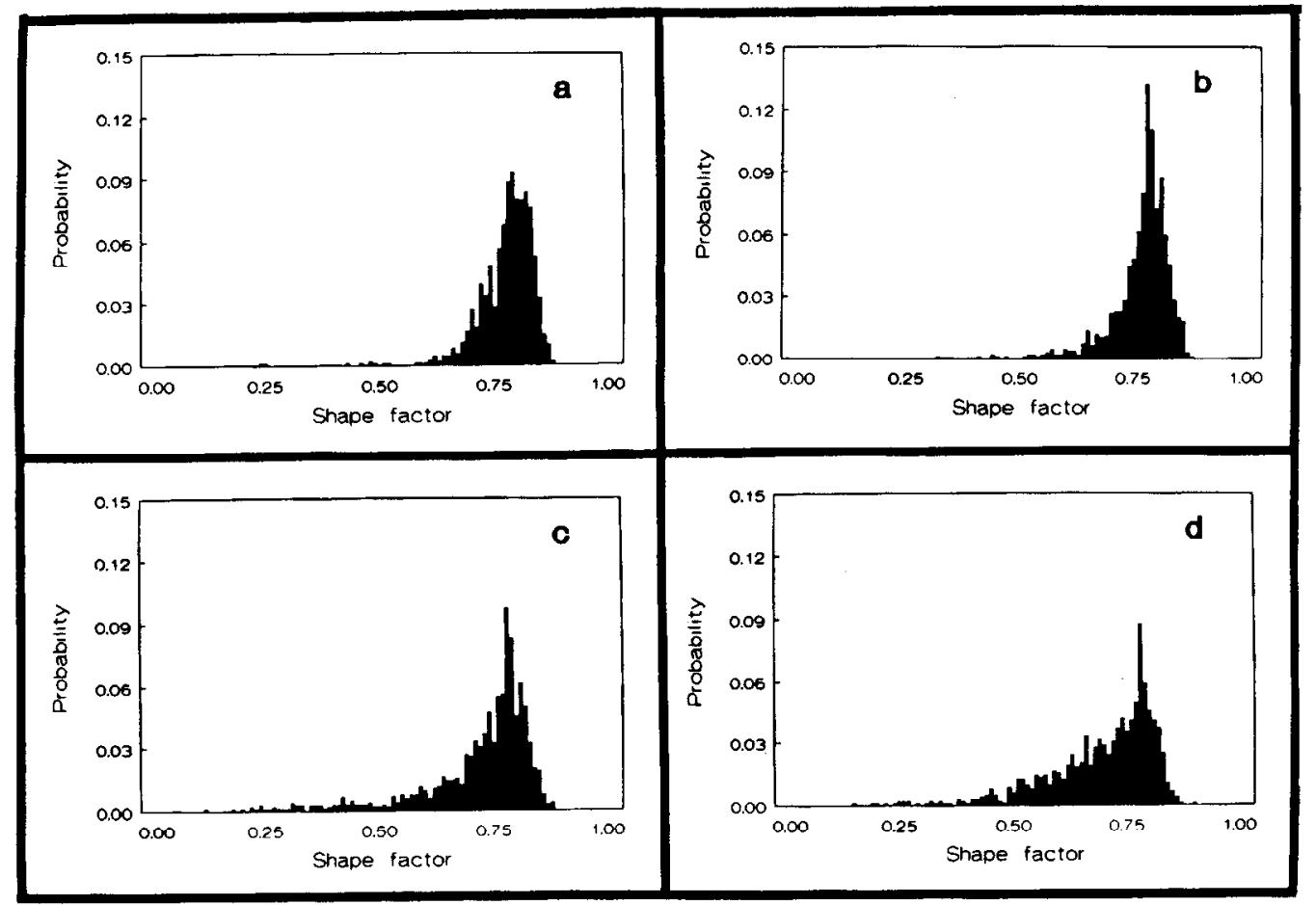

Fig. 2. Probability distribution of shape factor for container media components: (a) coarse sand, (b) fine sand, (c) coarse pine bark, and (d) fine pine bark.

particles are mainly spherical.

Figure 3a shows simulated porosity for a system of uniformsized spheres vs. Monte Carlo time. Once all particles have fallen to the bottom of the container $(\approx 1000$ Monte Carlo steps), porosity does not change substantially; therefore, we chose 5000 Monte Carlo steps to perform our shrinkage simulations. Similarly (Fig. $3 b), 20$ min was chosen as the standard time for experimental bulk volume calculations (Pokorny and Henny, 1984). Figure 4a shows simulated shrinkage obtained by mixing spheres of radii 0.460 and 0.255 , which are the average mesh sizes divided by two. Although one usually takes the mean size of a sieved material to be the arithmetic mean of the mesh sizes of the two consecutive sieves (De Baker, 1983), in our case this underestimated shrinkage. When mixtures of coarse sand and fine sand were shaken for $20 \mathrm{~min}$ (Fig. $4 \mathrm{~b}$ ), the shrinkage curve was very similar to that where the materials were not shaken. Major changes in porosity (Fig. 3) do not appear to affect shrinkage substantially when different degrees of compaction are used (Fig. 4); thus, we should not expect differences between experimental and simulated shrinkage due to compaction.

Actual radius size distribution for the four materials was broad (Fig. 5). The average radii were $0.625548 \mathrm{~mm}$ (coarse) and 0.196172 (fine) for sand and 0.635078 (coarse) and 0.340833 (fine) for pine bark, values that do not correspond to the average mesh size divided by two. The simulation was repeated for a mixture of coarse and fine sand (monosize spheres), with radii equal to the actual average radii of sand particles (Fig. 6a). Simulated shrinkage was higher than the experimental with some of the mixture combinations.

When the simulation was repeated by generating spheres that had the actual size distribution of sand particles (Fig. 6b), computer-generated results agreed with experimental results. In this instance, the bulk volume : particle volume ratio was not necessarily constant within large or small particles, because a distribution of different particle sizes can result in differences in porosity. When bulk volumes of large and small particles were compared,

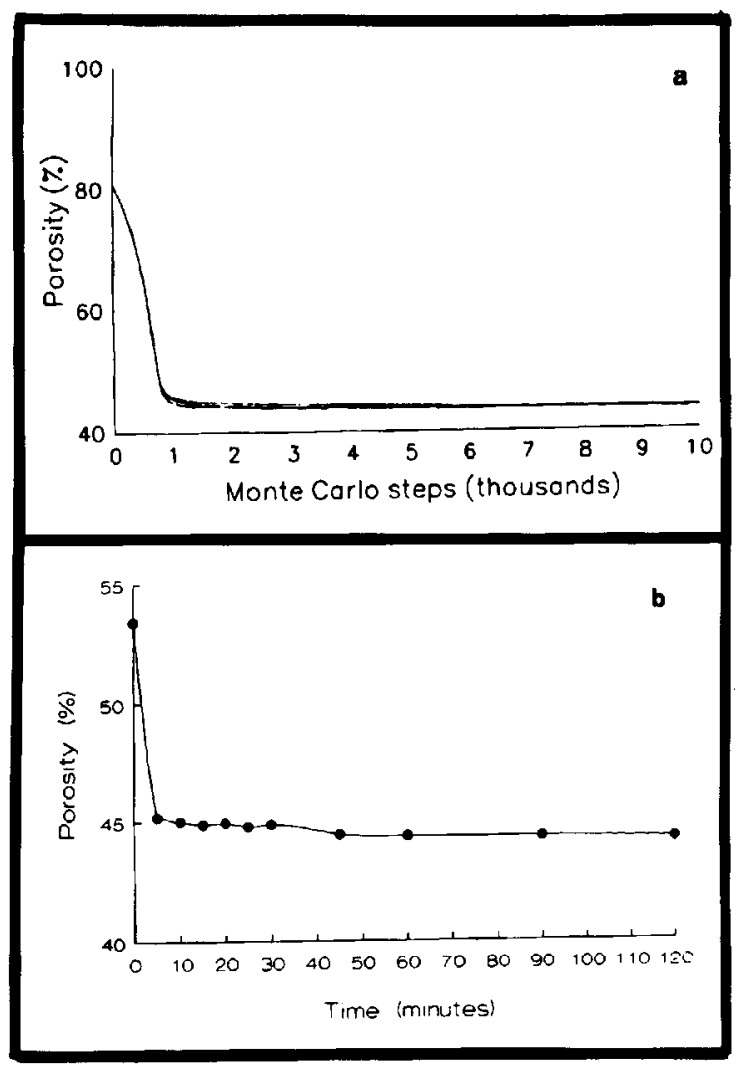

Fig. 3. Evolution of porosity (percent by volume) with time. (a) Computer simulated porosity vs. Monte Carlo time for a system of 1000 uniform spheres of radius $0.5 \mathrm{~mm}$ and a $10 \times 10 \times 30-\mathrm{mm}$ container. Porosity at time zero is that of the container with unpacked spheres situated at random, before the settling procedure (Monte Carlo process) starts; at time 10,000, all spheres are randomly packed at the bottom of the container. (b) Experimental porosity vs. time on a mechanical wrist-action shaker for fine sand. The initial porosity is that of a cylinder filled by hand with sand, before shaking starts; after shaking, sand particles settle to a constant volume. 


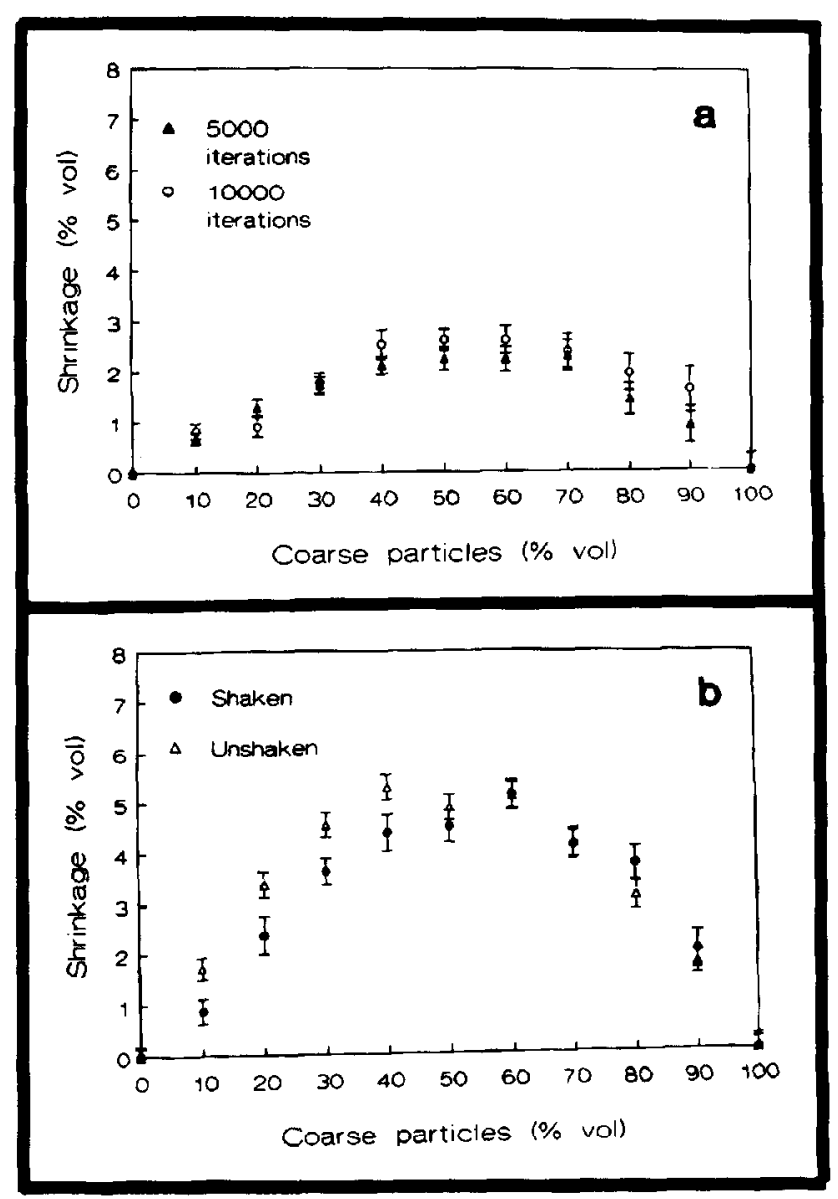

Fig. 4. Dependence of shrinkage on packing density. (a) Computer-simulated shrinkage curves of a mixture of two uniform sizes of spheres (radii, 0.460 and $0.255 \mathrm{~mm}$ ) at two different Monte Carlo time steps (container size $=5 \times 5 \times 15$ $\mathrm{mm}$ ). (b) Experiment-based shrinkage curves of mixtures of coarse and fine sand shaken for $20 \mathrm{~min}$ and not shaken. they were not statistically different; thus, we could use the same approach as with the uniform-sized mixtures. One must, nevertheless, take this into account when simulations are performed.

Computer simulation shows that a binary mixture of particle distributions shrinks less than uniform spheres of the same mean size as the distribution. This result agrees with published experimental data (Sohn and Moreland, 1968). Therefore, in simulating a container medium system, particle size distribution is a prime factor when establishing a relationship between the simulated and the experimental systems.

Relative particle sizes affect the degree of shrinkage, but the shape of the shrinkage curve is unaffected. Our experimental results show that maximum shrinkage in a binary mixture is obtained when the coarse component is added to the mixture in a proportion ranging from $50 \%$ to $70 \%$ of the additive volume of coarse and fine components. The maximum shrinkage, however, might also depend on other characteristics (e.g., shape, composition) of the materials being mixed. This consideration is important in substrate formulation, because maximum shrinkage indicates minimum porosity (interparticle porosity decreases as shrinkage increases) or "threshold proportion" (Spomer, 1974). Therefore, care should be exercised when mixing materials of different sizes to increase porosity, because the opposite effect can be attained. Internal porosity (Pokorny, 1987) of the mixed materials can, in some cases, counteract this effect; therefore, one must be aware of the specific characteristics of the materials being mixed.

Allen (1985) reported unequal porosities for materials of different shapes; porosity was higher when shapes differed from spherical. When materials were rod-like, porosity by volume of a random loose packing was $55 \%$, whereas values reported for random loose packing of uniform spheres were $\approx 40 \%$ to $43 \%$ (Scott and Kilgour, 1969). One should distinguish here between two types of porosity. External porosity may change with particle shape, while internal porosity depends exclusively on the nature of the mixed material. Maximum external porosity for spherical particles is obtained when all particles are the same size, and while the size of uniform

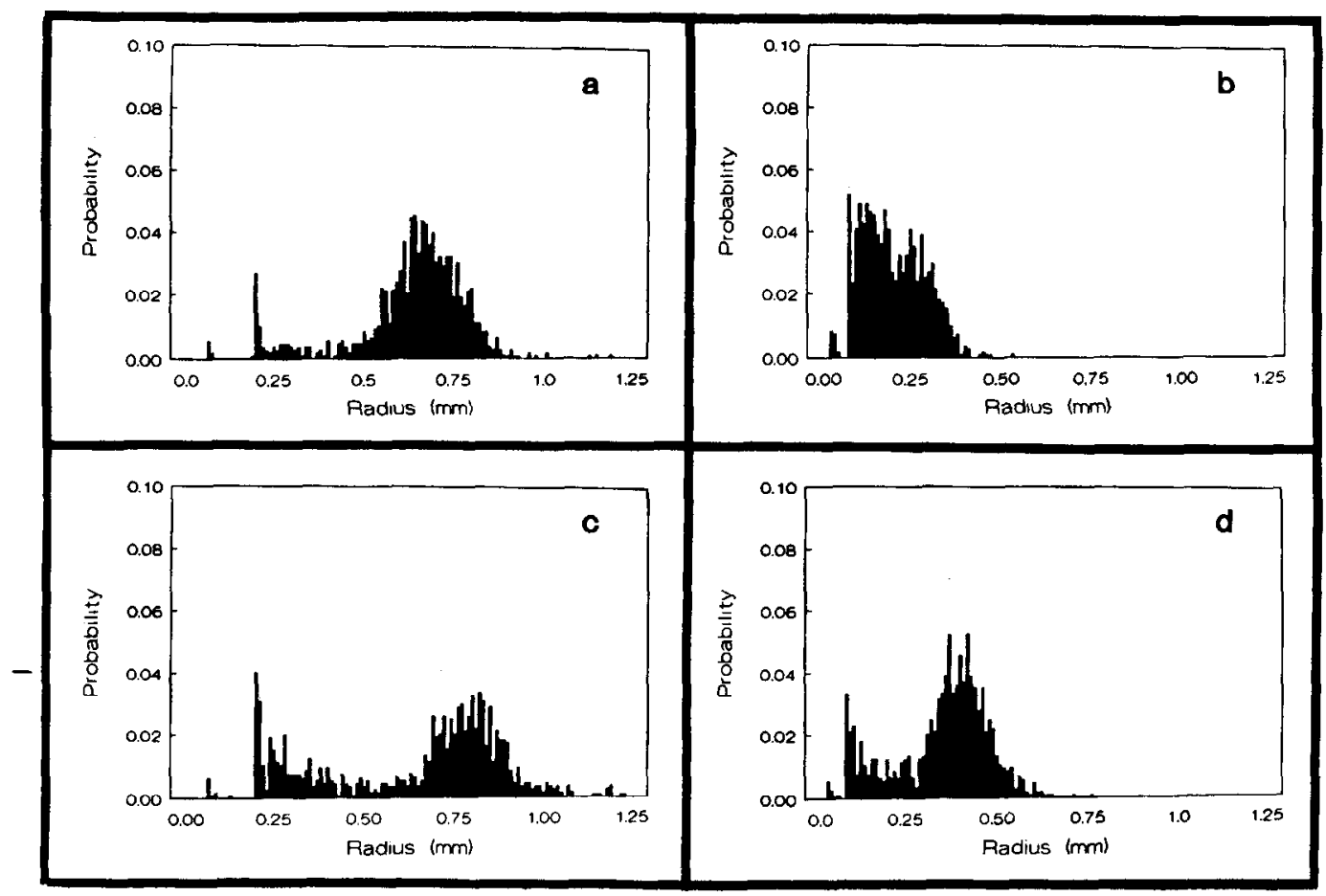

Fig. 5. Probability distribution of radii of container media components for (a) coarse sand, (b) fine sand, (c) coarse pine bark, and (d) fine pine bark. 


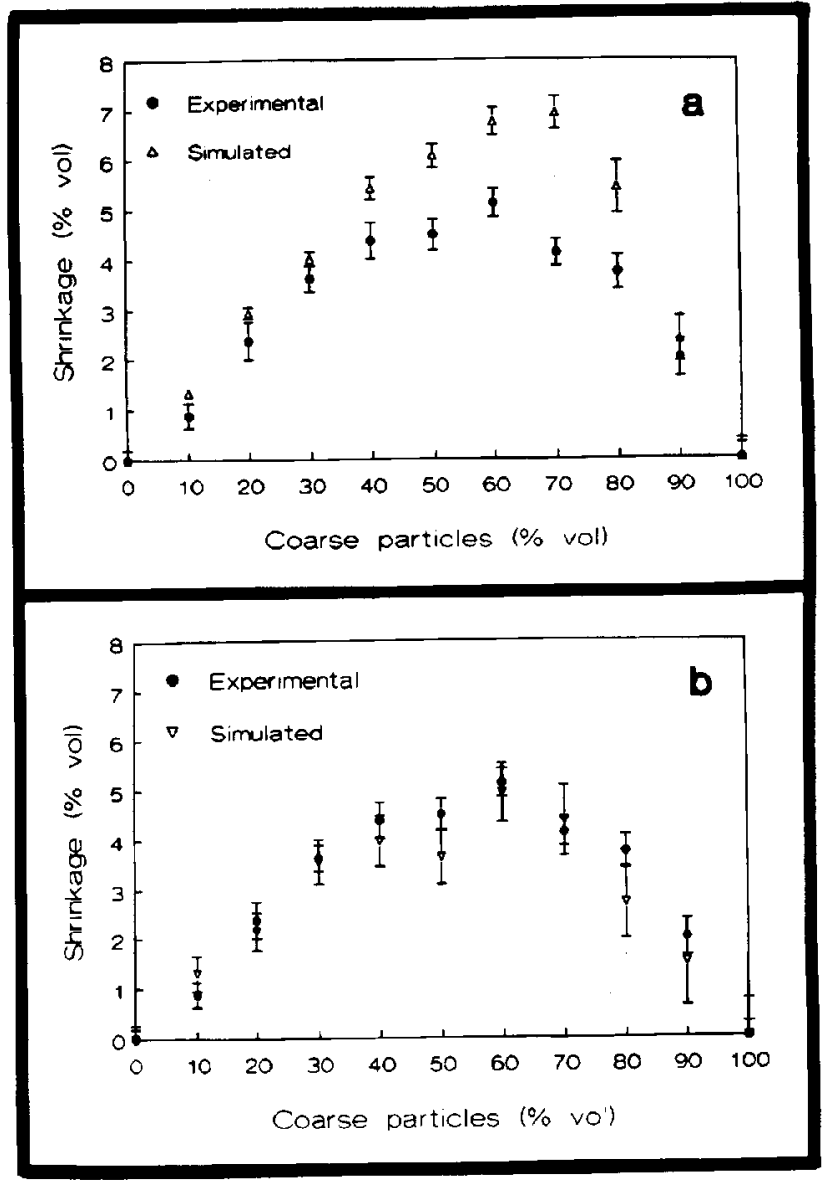

Fig. 6. Computer-generated vs. experiment-based shrinkage curves for a container medium mixture of coarse and fine sand. The simulated curves were obtained from (a) mixing two systems of monosize spheres with the same average radii as sand particles used in the experiment, or (b) mixing two systems of spheres that had, respectively, the same probability distribution of radii as the sand particles used in the experiment (container size $=6 \times 6 \times 18 \mathrm{~mm}$ ).

spherical particles may affect pore size, it does not affect total external porosity. Uniformity and actual pore size would account for the improvement in air porosity when a mixture contains a proportion of large particles that is greater than that which generates the maximum shrinkage.

Monte Carlo simulation has proven useful for reproducing the physical phenomenon of volume shrinkage of mixed container media particles. The possibility of easily changing variables, such as size or size distribution, makes it a valuable tool in understanding container media phenomena.

\section{Literature Cited}

Allen, J.R.L. 1985. Principles of physical sedimentology. George Allen \& Unwin, London.

Bodman, G.B. and G.K. Constantin. 1965. Influence of particle size distribution in soil compaction. Hillgardia 35:567-591.

Burés, S., D.P. Landau, A.M. Ferrenberg, and F.A. Pokorny. 1993. Monte Carlo computer simulation in horticulture: A model for container media characterization. HortScience. (In press.)

Burés, S. and F.A. Pokorny. 1991. Equation for estimating shrinkage in binary mixtures of container media. HortScience 26:1087.

Dallavalle, J.M. 1948. Micromeritics. 2nd ed. Pitman, London.

Davis, I.L. and R.G. Carter. 1990. Random particle packing by reduced dimension algorithms. J. Applied Phys. 67:1022-1029.

De Backer, L.W. 1983. Characterization of some retention and transfer properties of granular materials in relation with geometrical and liquid properties, p. 399-414. In: M. Shahinpoor (ed.). Advances in the mechanics and the flow of granular materials. Trans Tech., FR Germany \& Gulf, Houston.

Handreck, K.A. 1983. Particle size and the physical properties of growing media for containers. Commun. Soil Sci. Plant Anal. 14:209-222.

Haughey, D.P. and S.G. Beveridge. 1969. Structural properties of packed beds-A review. Can. J. Chem Eng. 47:130-140.

Nash, M.A. and F.A. Pokorny. 1990. Shrinkage of selected two-component container media. HortScience 25:930-931.

Pokorny, F.A. 1987. Available water and root development within the micropores of pine bark particles. J. Environ. Hort. 5:89-92.

Pokorny, F.A. and B.K. Henny. 1984. Construction of a milled pine bark and sand potting medium from component particles. I. Bulk density: A tool for predicting component volumes. J. Amer. Soc. Hort. Sci. 109:770-773.

Richards, D., M. Lane, and D.V. Beardsell. 1986. The influence of particle-size distribution in pinebark : sand : brown coal potting mixes on water supply, aeration and plant growth. Scientia Hort. 29:1-14.

Scott, G.D. and D.M. Kilgour. 1969. The density of random close packing of spheres. Brit. J. Applied Phys. (J. Phys. D) 2:863-866.

Sohn, H.Y. and C. Moreland. 1968. The effect of particle size distribution on packing density. Can. J. Chem. Eng. 46:162-167.

Spomer, L.A. 1974. Optimizing container soil amendment: The "threshold proportion" and prediction of porosity. HortScience 9:532-533.

Visscher, W.M. and M. Bolsterli. 1972. Random packing of equal and unequal spheres in two and three dimensions. Nature 239:504-507.

Westman, A.E.R. 1936. The packing of particles: Empirical equations for intermediate diameter ratios. Amer. Ceramic Soc. J. 19:127-129.

Westman, A.E.R. and H.R. Hugill. 1930. The packing of particles. Amer. Ceramic Soc. J. 13:767-779. 